\title{
Primary Mediastinal Pleomorphic Rhabdomyosarcoma: A Case Report
}

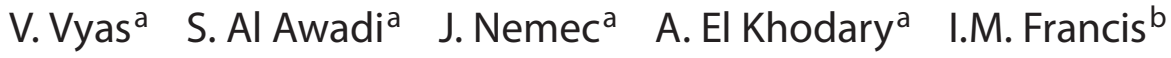 \\ K.C. Muralidharan ${ }^{a}$ M.D. Delvadiya ${ }^{a}$ \\ Departments of a Medical Oncology and ${ }^{b}$ Pathology, Kuwait Cancer Control Centre, Kuwait
}

\section{Key Words}

Rhabdomyosarcoma $\cdot$ Mediastinal germ cell tumour •

Teratoma

\begin{abstract}
Objective: To report a rare case of pleomorphic rhabdomyosarcoma which occurred in the mediastinum of a 34year-old man. Clinical Presentation and Intervention: A young male labourer presented with dyspnoea on exertion. A large mediastinal mass was detected on chest CT scan. The chest surgeons advised against open biopsy. His $\alpha$-fetoprotein was 22,000 IU/l; based on this the diagnosis of a germ cell tumour was made and the patient was treated with a bleomycin/etoposide/cisplatin regimen. He left for his native country where an open biopsy from the mediastinum was taken and reported as pleomorphic rhabdomyosarcoma. He was given five courses of chemotherapy with doxorubicin, etoposide, and ifosfamide with mesna protection without much relief. The inoperable disease occupied the whole of the right chest and mediastinum. The enormous size of the radiation field made radiotherapy prohibitive. Finally, the patient opted for symptomatic treatment and left for his native place. Conclusion: This case is presented because of its difficulty in management and rarity.
\end{abstract}

Copyright $\odot 2008$ S. Karger AG, Basel

\section{Introduction}

Germ cell tumours are rare and generally occur in the testes and ovaries, as well as in the mediastinum or retroperitoneum. Mediastinal germ cell tumours in adult patients represent $2-5 \%$ of all germ cell tumours and only $1-10 \%$ of mediastinal tumours $[1,2]$. Germ cell tumours are observed both in children below 7 years of age and in adults from 15 to 35 years of age [3]. They are more frequent in male than female patients. Primary mediastinal germ cell tumours have the same histological pattern as their gonadal counterparts. The prognosis of mediastinal germ cell tumours is poor, only $30 \%$ of patients being cured [1].

\section{Case Report}

Mr. H.R. was a 30-year-old labourer and ex-smoker of water pipe; he had ceased smoking a year before. No medical comorbidity was indicated. In August 2005, he presented with a history of dry cough, shortness of breath and right pleuritic pain. On examination, no peripheral lymph nodes were palpable, pulse was $117 / \mathrm{min}$ and regular, blood pressure was $140 / 90 \mathrm{~mm} \mathrm{Hg}$ and $\mathrm{SpO}_{2} \%$ was $95 \%$ at room air. The trachea was shifted to the left; there was right-sided dullness and decreased air entry on the right side. 
Chest X-ray (posterior-anterior view) showed mediastinal widening and a huge mass occupying the right hemithorax. A computerised axial tomography scan of the chest showed a $13-\mathrm{cm}$ right-sided anterior mediastinal mass, inhomogeneous attenuation with scattered flecks of calcification, occupying almost the whole right hemithorax and compressing the right lung posteriorly with mediastinal shift to the left side. No rib changes, right pleural effusion or lymphadenopathy were seen. Radiological impression was that of a right anterior mediastinal mass, possibly germ cell tumour or thymoma.

Diagnostic aspiration of $150 \mathrm{ml}$ fluid was negative for malignant cells and acid-fast bacilli. Bronchoscopy showed normal vocal cords, sharp carina, marked extraluminal compression of the right main bronchus and normal mucosa. Broncho-alveolar lavage for cytology yielded inadequate material but the culture was negative for acid-fast bacilli. Fine-needle aspiration cytology and core biopsy from the mass showed only non-diagnostic totally necrotic material. Ultrasound of the testis did not reveal any mass, and $\alpha$-fetoprotein (AFP) was 22,000 IU/l.

The chest surgeons advised against open biopsy. Subsequently, in September 2005, the patient was seen in the cancer centre with clinical diagnosis of mediastinal germ cell tumour and was started on combination chemotherapy with a bleomycin/etoposide/ cisplatin (BEP) regimen. The level of AFP had increased to 38,342 IU/l, which was the baseline for chemotherapy. After the first course of chemotherapy, the patient left for his native country (without letting his physician know and without a medical report) where an open mediastinal biopsy was performed.

The entire specimen was processed for histological evaluation and multiple tissue sections covering the entire tissue sample were examined. Microscopic examination revealed a rather pleomorphic spindle cell neoplasm with marked nuclear atypia and prominent mitotic activity. The tumour exhibited frank rhabdomyoblastic differentiation despite the rather pleomorphic nature. Careful search was done for any yolk sac differentiation. A panel of immunostains showed unequivocal positivity for myoglobin, desmin and vimentin, strongly supporting rhabdomyoblastic differentiation in this neoplasm. The tumour did not reveal any expression of cytokeratin, CD 117, AFP, or S100 protein. In view of the fact that the tissue provided to the pathology lab was only a small portion of a large mass, with the significantly elevated AFP in the patient's serum, evidence of yolk sac differentiation could not be totally ruled out despite the morphological finding in this case. The pathology indicated rhabdomyoblastic differentiation in a germ cell tumour (fig. 1). The patient was given five cycles of chemotherapy with doxorubicin, etoposide, and ifosfamide with mesna protection without much relief. Rather, his dyspnoea increased, and he developed right-sided empyema requiring intercostal tube drainage. The patient returned to Kuwait and at this time, his AFP had decreased to 6,000 IU/l. CT scan of the chest showed a mass occupying the anterior mediastinum extending into the right lung, with solid and cystic areas.

The patient was restarted on BEP chemotherapy. His AFP at this time was 20,498. After two cycles of chemotherapy his AFP came down to 2,000. A repeat CT scan chest showed a persistent right chest heterogeneous mass. Surgeons and radiation oncologists were consulted. Surgeons advised against operation, and radiation oncologists were not able to proceed with palliative radiotherapy due the extensive radiation field. Finally, the patient decided to stop active treatment and went back to his native place.

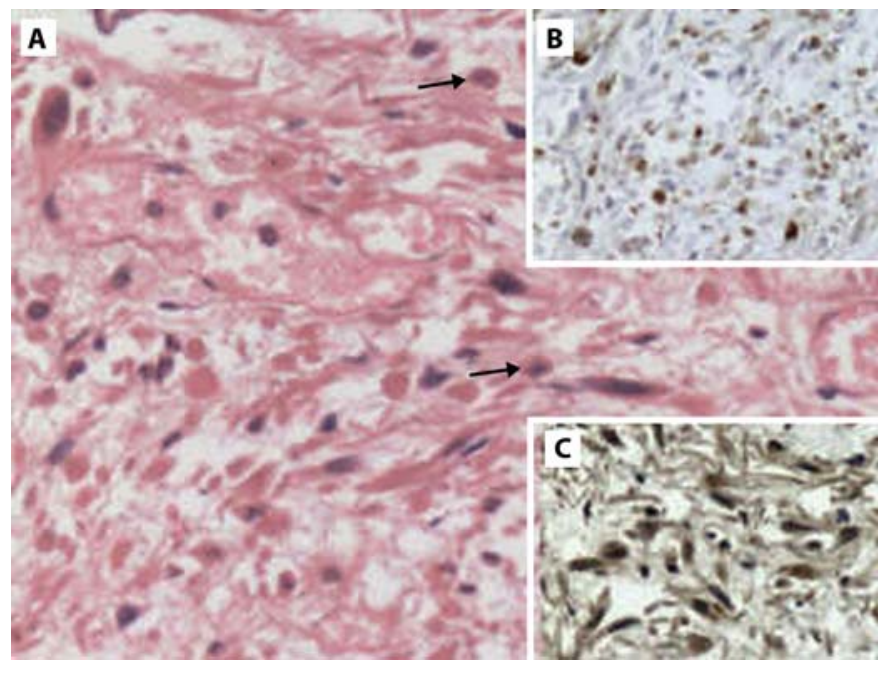

Fig. 1. Photomicrograph of mediastinal lesion showing a pleomorphic malignant tumour with evident rhabdomyoblastic cell differentiation (arrows). Tumour cells showed expression of both desmin (A) and myoglobin (C). A HE, 5- $\mu$ m-thick paraffin sections. $\times 200$. B, C Immunohistochemistry using ABC method against desmin and myoglobin antigens.

\section{Discussion}

Mediastinal germ cell tumours exhibit a poor prognosis. Only $30-40 \%$ of patients are cured [2]. A non-germinal malignant tumour developing within a germ cell tumour is an extremely rare event [3] and was first observed in teratomatous elements after chemotherapy in testicular cancer $[4,5]$. These malignant transformations are generally sarcomas and mostly rhabdomyosarcomas [6].

Occurrence of a sarcomatous component in a mediastinal teratoma has no specific initial features. Diagnosis can be fortuitous at the time of histopathological examination of a tumour biopsy or after tumour resection or, more frequently, an unusual outcome with chemotherapy in a presumed non-teratomatous germ cell tumour attracting attention [3].

Mediastinal rhabdomyosarcomas are most often resistant to chemotherapy as in our patient. They have poorer prognosis, worse than their gonadal counterparts $[6,7]$, with a short survival of $<2$ years. Patients usually die due to regional and distant metastases $[3,6]$. At present, there is no standard chemotherapy for adult rhabdomyosarcoma. The paediatric protocols are often not effective in adults and there is no chemotherapy effective for both germinal and non-germinal components. The 
most important prognostic factors in adult rhabdomyosarcoma are age, tumour size, extent of disease, and resection [7]. The median survival in a population of 84 patients was 22 months despite aggressive multimodality treatment.

It is noteworthy that in our case, as in others, the germ cell component of the tumour was very chemosensitive as indicated by a drop in AFP values after treatment with chemotherapy. However, rhabdomyosarcoma was refractory to the chemotherapy regimen considered effective in this tumour type. Previous reports [1, 2, 4, 6, 8-11] have shown that this tumour demonstrates a poor prognosis (only 1 out of 16 patients is cured). As reported by Gonzalez-Vela et al. [8], out of the 15 patients with mediastinal germ cell tumours, 4 with a sarcoma component died of the disease, but 6 of 11 patients without a sarcoma component survived.

In another series of teratoma with malignant transformation in which 5 out of 12 patients had undifferentiated rhabdomyosarcoma, chemotherapy was administered according to the somatic differentiation and the conclusion was that chemotherapy for malignant transformation limited to a single cell type may result in major responses and long-term survival in selected patients and that local therapy after chemotherapy is an important component of treatment to achieve maximum response [12].

Experience proves that surgery is a very important part of teratoma management; complete surgical resection appears to be the most important prognostic feature.

\section{Conclusion}

Because of the risk of a sarcomatous component in mediastinal germ cell tumour and lack of an effective chemotherapy treatment, resection of residual masses should always be considered after first-line cisplatin-based chemotherapy.

\section{References}

1 Omezzine N, Khouatra C, Larivé S, Freyer G, Isaac-Pinet S, Gérinière L, Droz JP, Souquet PJ: Rhabdomyosarcoma arising in mediastinal teratoma in an adult man: a case report. Ann Oncol 2002;13:323-326.

-2 Dulmet EM, Macchiarini P, Suc B, Verley JM: Germ-cell tumors of the mediastinum. Cancer 1993;72:1894-1901.

-3 Moran CA, Suster S: Primary germ-cell tumors of the mediastinum: analysis of 322 cases with special emphasis on teratomatous lesions and a proposal for histopathologic classification and clinical staging. Cancer 1997;80:681-690.

-4 Motzer RJ, Amsterdam A, Prieto V, Sheinfeld J, Murty VVVS, Mazumdar M, Bosl GJ, Chaganti RSK, Reuter VE: Teratoma with malignant transformation: diverse malignant histologies arising in men, with germcell tumors. J Urol 1998;159:133-138.
5 Ahmed T, Bosl GJ, Hadju SI: Teratoma with malignant transformation in germ-cell tumors in men. Cancer 1985;56:860-863.

-6 Ulbright TM, Loehrer PJ, Roth LM, Einhorn LH, Williams SD, Clark SA: The development of non-germ-cell malignancies within germ-cell tumors: a clinicopathologic study of 11 cases. Cancer 1984;54:1824-1833.

$\checkmark 7$ Kattan J, Culine S, Terrier-Lacombe MJ, Theodore C, Droz JP: Paratesticular rhabdomyosarcoma in adult patients: 16-year experience at Institut Gustave-Roussy. Ann Oncol 1993;4:871-875.

$>8$ Gonzalez-Vela JD, Savage PD, Manivel JC, Torkelson JL, Kennedy BJ: Poor prognosis of mediastinal germ-cell cancers containing sarcomatous components. Cancer 1990;66: 1114-1116.
9 Caballero C, Gomez S, Matias-Guiu X: Rhabdomyosarcomas developing in association with mediastinal germ-cell tumours. Vichows Archiv A Pathol Anat 1992;420: 539-543.

10 Rebishung C, Fizari K, Daban A: Germ-cell tumours containing non germ-cell neoplasms: the so-called teratoma with malignant transformation (TMT). Ann Oncol 1998(suppl 4):abstr 281P.

11 Fizazi K, Culine S, Droz JP, Kramar A, Theodore C, Ruffie P, Chevalier TL: Primary mediastinal non seminomatous germ-cell tumors: results of modern therapy including cisplatin based chemotherapy. J Clin Oncol 1998;16:725-732.

12 Donadio AC, Motzer RJ, Bajorin DF, Kantoff PW, Sheinfeld J, Houldsworth J, Chaganti RS, Bosl GJ: Chemotherapy for teratoma with malignant transformation. J Clin Oncol 2003;21:4285-4291. 